\title{
Molecular Typing of Methicillin-Resistant Staphylococcus aureus (MRSA) Strains Isolated in Two Metropolitan Areas of São Paulo State, Southeast Brazil
}

\begin{abstract}
Gisele Gentile Cury¹, Cristiane Mobilon², Eliana Guedes Stehling², Marcelo Lancellotti ${ }^{2}$, Marcelo de Carvalho Ramos ${ }^{1}$, Roberto Martinez $^{3}$, Marcelo Brocchi ${ }^{2}$ and Wanderley Dias da Silveira ${ }^{2}$

${ }^{1}$ Department of Internal Medicine, School of Medicine, Unicamp; ${ }^{2}$ Department of Microbiology and Immunology, Biology Institute, Campinas State University, Campinas (Unicamp), SP; ${ }^{3}$ Department of Internal Medicine; School of Medicine; São Paulo State University at Ribeirão Preto-USP, Ribeirão Preto, SP; Brazil
\end{abstract}

\begin{abstract}
One hundred and fifty-one methicillin-resistant Staphylococcus aureus (MRSA) strains have been isolated from patients admitted in tertiary care hospitals in two metropolitan areas (Campinas City and Ribeirão Preto City) in the southeast region of Brazil and analyzed through PCR-based techniques [(PCR amplification of spa, coa, and housekeeping genes ( $\operatorname{arcC}$, aroE, gmk, pta, tpi, yqiL)] and further restriction fragment typing of coa and of housekeeping genes. The heterogeneity of spa gene was determined directly by agarose gel electrophoresis migration. The results obtained indicate the existence of three (A, B, C) main clusters. Since the strain distribution in these three clusters is much characteristic, it denotes the existence of three main clones. All strains isolated in Campinas were grouped in clusters A and B, while most of the strains isolated in Ribeirão Preto were grouped in cluster $C$. This distribution denotes the existence of different founder strains that undergo independent genetic variability. The strains considered representative of the Brazilian Epidemic Clone (BEC) were categorized as cluster $A$. These results indicate a possible higher variability among Brazilian MRSA strains than currently described and indicate that the techniques herein used can be used as an alternative to Pulsed Field Gel Electrophoresis (PFGE).
\end{abstract}

Key-Words: MRSA, nosocomial, molecular characterization, PCR.

Methicillin-resistant strains of Staphylococcus aureus (MRSA) have become one of the most significant nosocomial pathogens worldwide, being capable of causing an wide range of hospital infections and clinical syndromes associated with severe diseases, including bacteremia, pneumonia, endocarditis, septic arthritis, osteomyelitis, and deep abscess formation [1]. As MRSA strains can disseminate very rapidly, it is necessary to implement effective monitoring programs for the identification and control of epidemic strains [2].

Studies have revealed a highly clonal population, with low variability, between MRSA strains, consistent with the view that $S$. aureus, unlike some of the freely recombining pathogenic species such as Streptococcus pneumoniae [3], Neisseria meningitidis [4], and Helicobacter pylori [5] is not naturally transformable [6] presenting, then, a low number of clones.

One of these MRSA described clones, the Iberian MRSA, first identified as the dominant clone in a major MRSA disease outbreak in a hospital in Spain in 1989 [7] was subsequently detected in at least eight Portuguese hospitals [8] as well as in hospitals in Western Scotland, United Kingdom, Italy, Belgium, and Germany [9], and in one hospital in the USA [10]. A second multiresistant clone (Brazilian Epidemic clone - BEC) was

Received on 20 February 2009; revised 29 May 2009.

Address for correspondence: Dr. Wanderley Dias da Silveira. Department of Microbiology and Immunology, Biology Institute, Campinas State University UNICAMP. Rua Monteiro Lobato, s/n. PO BOX 6109 CEP 13083-862 - Campinas - SP- Brazil, Phone:+55 (19) 3788-6272. Fax: +55 (19) 3521-6276. E-mail: wds@unicamp.br.

The Brazilian Journal of Infectious Diseases 2009;13(3):165-169. (C) 2009 by The Brazilian Journal of Infectious Diseases and Contexto Publishing. All rights reserved. shown to be widely spread in Brazilian hospitals several thousands of kilometers apart [11] and later it was also associated with infections in Portugal, Argentina, Uruguay, Czech Republic, and Canada [12-15]. Several other studies [16-18] in Brazil, using pulsed-field gel electrophoresis (PFGE) technique, to analyze Brazilian MRSA strains, also indicated a low variability between strains.

In this work, to assess the existing genomic variability of 151 methicillin-resistant Staphylococcus aureus (MRSA) strains recovered from patients attended in two tertiary care hospitals, at the Medical School Hospital of Campinas State University (UNICAMP) in Campinas City and at the Medical School Hospital of São Paulo University in Ribeirão Preto City (FCMRP-USP), both cities located 155.34 miles apart in the Southeast region of Brazil, was used a combination of PCR-based techniques [(PCR amplification of spa and coa genes and housekeeping genes ( $\operatorname{arc\mathrm {C}}$, aroE, gmk, pta, tpi, yqiL)] with further restriction and fragment typing of the coa gene and housekeeping genes (RFLP), plus the direct determination of the heterogeneity of the spa gene by agarose gel electrophoresis migration $[3,6,19]$. This approach would be able to show a possible clonal variability in these MRSA strains that was not demonstred in previously published works [16-18].

\section{Material and Methods}

Strains

One-hundred and forty-five MRSA strains investigated in this work were recovered from patients in two Medical School Hospitals located in metropolitan areas of São Paulo State, Southeast Brazil between 2002 and 2005. Four strains (257A, 20COA, 256B, and 24COA) also obtained in the Medical 
School Hospital of Campinas State University were considered in this work as representative strains of a previously described work [20]. As control strains and for comparison sake in the clustering analysis, another 2 strains (BEC 9393; HC 562) [17] were used.

\section{Genomic DNA Extraction}

MRSA strains were grown overnight in LB medium [21] at $37^{\circ} \mathrm{C}$ and total DNA was extracted as described by Ausubel et al. [22].

PCR Amplification and Analysis of the Polymorphism of spa Gene

The amplification (Perkin Elmer ${ }^{\circledR}$ DNA Thermal Cycler 480 Waltham, Massachusetts, USA) of DNA fragments of the spa gene and the polymorphisms of the fragments were analyzed by agarose gel electrophoresis as described by Koreen et al. [23] (Figure 1A).

PCR Amplification and Analysis of the Polymorphism of the coa Gene

The amplification of DNA fragments, by PCR, of the coa gene and further restriction with endonucleases and agarose gel electrophoresis followed the protocol described by Montesinos et al. [24] using a Perkin Elmer® DNA apparatus (Thermal Cycler 480 Waltham, Massachusetts, USA) (Figure 1B).

PCR Amplification of the Housekeeping Genes and Polymorphism Analysis

Amplification of the housekeeping genes (Perkin Elmer ${ }^{\circledR}$ DNA Thermal Cycler 480 Waltham, Massachusetts, USA) and restriction-generated fragments were performed as described by Diep et al. [25]. Fragments polymorphisms were analyzed by agarose gel electrophoresis as described by Sambrook et al. [21] (Figure 2).

\section{Genetic Similarity and Statistical Analysis}

The genomic similarity between strains was assessed throughout the construction of a similarity dendrogram generated by the NTSys software (UPGMA algorithm) using the combination of all generated fragments obtained by all PCR-based techniques [26] (Figure 3).

\section{Results and Discussion}

The results herein obtained by the combination of all the methods indicate the existence of three main clusters (A; B; C) of MRSA in these two metropolitan areas (Figures 1 and 2). Cluster A comprised 29.5\% $(n=45)$ of the strains, and contained the representative BEC strain (BEC9393) and strain HC562, which were previously studied [16] and also contained four strains isolated in the metropolitan Ribeirão Preto. The second cluster (B) comprised most of the strains (57.6\%; $n=90)$, and with the exception of only one (1RP), it contained only strains isolated in Campinas city metropolitan area. The third cluster
(C) $(n=21)$ contained only strains obtained in metropolitan Ribeirão Preto. Cluster $\mathrm{C}$ had a smaller genetic proximity (approximately 10\%) with clusters A and B, which had a greater genetic proximity (aproximately 25\%). Inside clusters A and B there were strains that showed $100 \%$ of similarity with others strains (Table 1). This result suggests the presence of clones among the studied strains. Independently of the strain numbers that were isolated in both metropolitan hospitals, it is possible to observe that: (i) strains from one geographical area tend to evolve forming predominant successful clones responding to specific selective pressures; (ii) strains from one geographical area can present genetic proximity with strains from another geographical area; (iii) just a few cluster of strains, with variable genetic similarity, are observed; (iv) inside these clusters there are real strain clones and (v) the $\mathrm{BEC}$ representative strain is not the predominant one among all the isolated strains. These findings support the data published by Montesinos et al. [23].

Considering that the strains were obtained recently, from 2002 to 2005, the genetic profiles observed show that genetic divergent events occurred considerably fast. This genomic variability might be explained by selective local pressures that force horizontal genetic recombinant events, preserving certain successful "variable” genotypes. Previous studies have revealed a highly clonal population, consistent with the view that $S$. aureus, unlike the freely recombining pathogenic species Streptococcus pneumoniae [3], Neisseria meningitidis [4], and Helicobacter pylori [5], is not naturally transformable. In opposition, our data, indicating that MRSA has a genetic diversity, even if we are analyzing genes that should not diverge (housekeeping genes), support those published by Feil et al. [6] and Kuhn et al. [27] reporting that although recombination in $S$. aureus is uncommon, it can occur with sufficient frequency within and between loci to generate a very large number of allelic combinations. In summary, the results herein obtained indicate the possible existence of three main MRSA strains clusters in Southeast Brazil, all of them raised possibly due to local selective pressures and to genetic recombination events. This suggests that a high genetic variability would also exist in other Brazilian regions. The so-called Brazilian Epidemic Clone [15] would be one of the existing clones in Brazil, but others yet undetected must also exist and, finally, there are geneticaly similar strains in geographical areas located not so proximate which could arise either by the carriage of patients that seak for medical treatment in different centers, by the carriage of medical personal that exchange positions between these centers, or even by convergent evolution. Concluding, the study of MRSA strains by a sum of techniques like the ones employed in this work also demonstrates that Brazilian MRSA has a high genetic variability. These techniques may be used either before PFGE technique, or in place of PFGE, what would make the study of bacterial population in a fast and little expensive way an open possibility. 
Figure 1. Agarose (1.5\%) gel electrophoresis of PCR-amplified fragments spa and coa genes. (1) Molecular weight 1Kb marker; (A) amplified spa fragments; (B) coa fragments obtained after PCR; (C) coa fragments obtained after digestion with enzyme Hae III; (D) coa fragments obtained after digestion with enzyme Alu I.

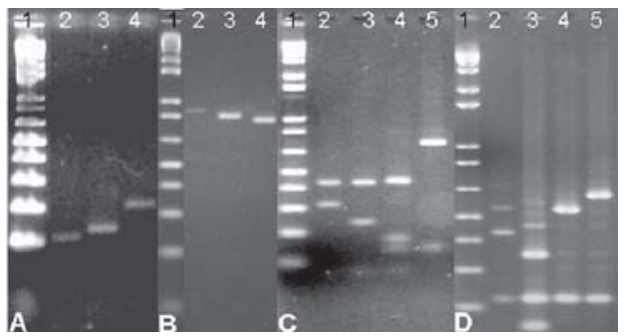

Figure 2. Agarose (1.5\%) gel electrophoresis of PCR-amplified fragments of housekeeping genes and fragments obtained after digestion with different restriction enzymes. (1) Molecular weight 1Kb marker; (A) locus aroE (enzyme CfoI); (B) locus yqiL (enzyme VspI); (C) locus pta (enzyme RsaI); (D) locus yqiL (enzyme DdeI); (E) locus tpi (enzyme BbuI);(F) locus tpi (enzyme MboI); (G) locus arcC (enzyme HinfI); (H) locus aroE (enzyme AluI); (I) locus gmk (enzyme CfoI).

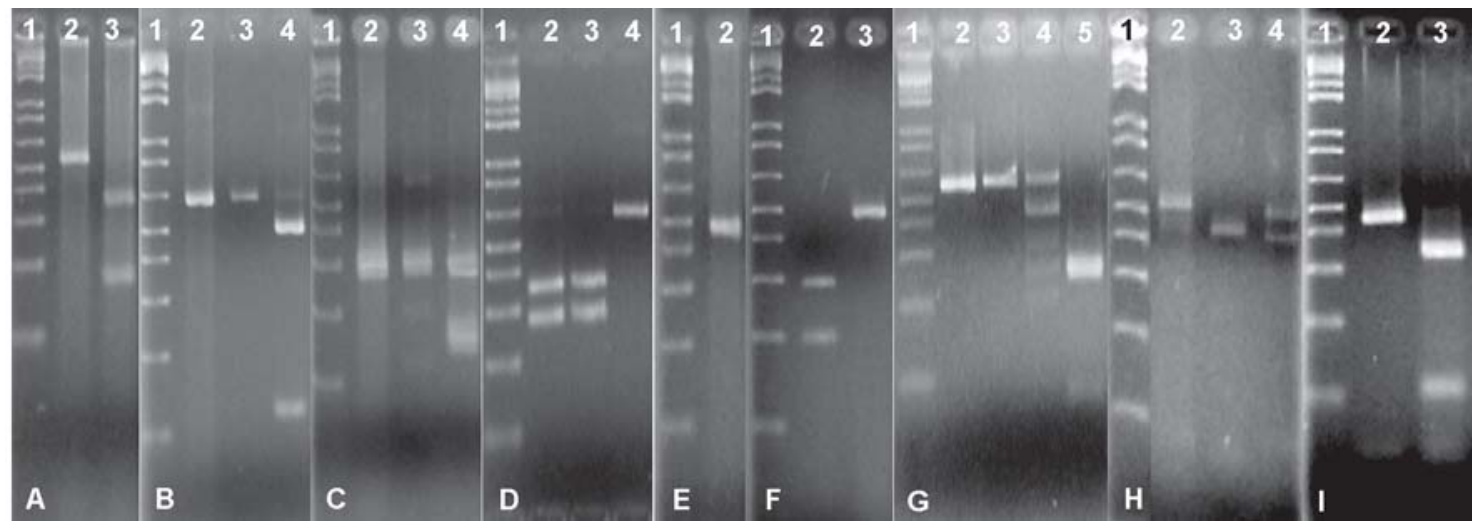

Table 1. Isolates with identical similarity and the cluster in which these isolates are grouped. All the isolates, except the ones named with RP, were obtained in the Medical School Hospital of Campinas State University. The isolates BEC 9393 and HC 562 were obtained in Rio de Janeiro.

\begin{tabular}{ccc}
\hline $\begin{array}{c}\text { Isolates represented } \\
\text { in the dendrogram }\end{array}$ & Isolates with 100\% of similarity & Cluster \\
\hline 7 & $14,35,72,131,157,185$ & $\mathrm{~A}$ \\
$257 \mathrm{~A}$ & $20 \mathrm{COA}$ & $\mathrm{A}$ \\
5 & $8,34,35,36,37,39,41,43,45,48,50,123,124,125,139,146,152,161,169,170,171,179$ & $\mathrm{~B}$ \\
4 & $46,102,107,109,122,142,150,164,167,168,172,184,187$ & $\mathrm{~B}$ \\
12 & $16,17,20,58,141$ & $\mathrm{~B}$ \\
106 & 130 & $\mathrm{~B}$ \\
180 & $18,135,140$ & $\mathrm{~B}$ \\
6 & $18,21,22,23,28,30,31,32,66,71,74,75,80,8,86,94,97,99,110,143,149,155,156 ; 173$ & $\mathrm{~B}$ \\
9 & 174 & $\mathrm{~B}$ \\
162 & 19 & $\mathrm{~B}$ \\
\hline
\end{tabular}

\section{Acknowledgments}

This work was supported by FAPESP (Fundação de Amparo à Pesquisa do Estado de São Paulo) grant No. 03/08407-0 and CAPES grant No. 832386/1999-5. The authors are thankful to Dr. Maria Clara Padoveze [20] from Campinas State University for the donation of isolates 257A, 20COA, 256B, and HC562, and to Dr.
Agnes M. Figueiredo [17] from Universidade Federal do Rio de Janeiro for the donation of isolates BEC 9393 and HC 562.

\section{References}

1. Ayliffe G.A.J. The progressive intercontinental spread of methicillin-resistant Staphylococcus aureus. Clin Infect Dis 1997;24(1):74-9. 
Figure 3. Dendrogram of genetic similarity (\%) of isolates.

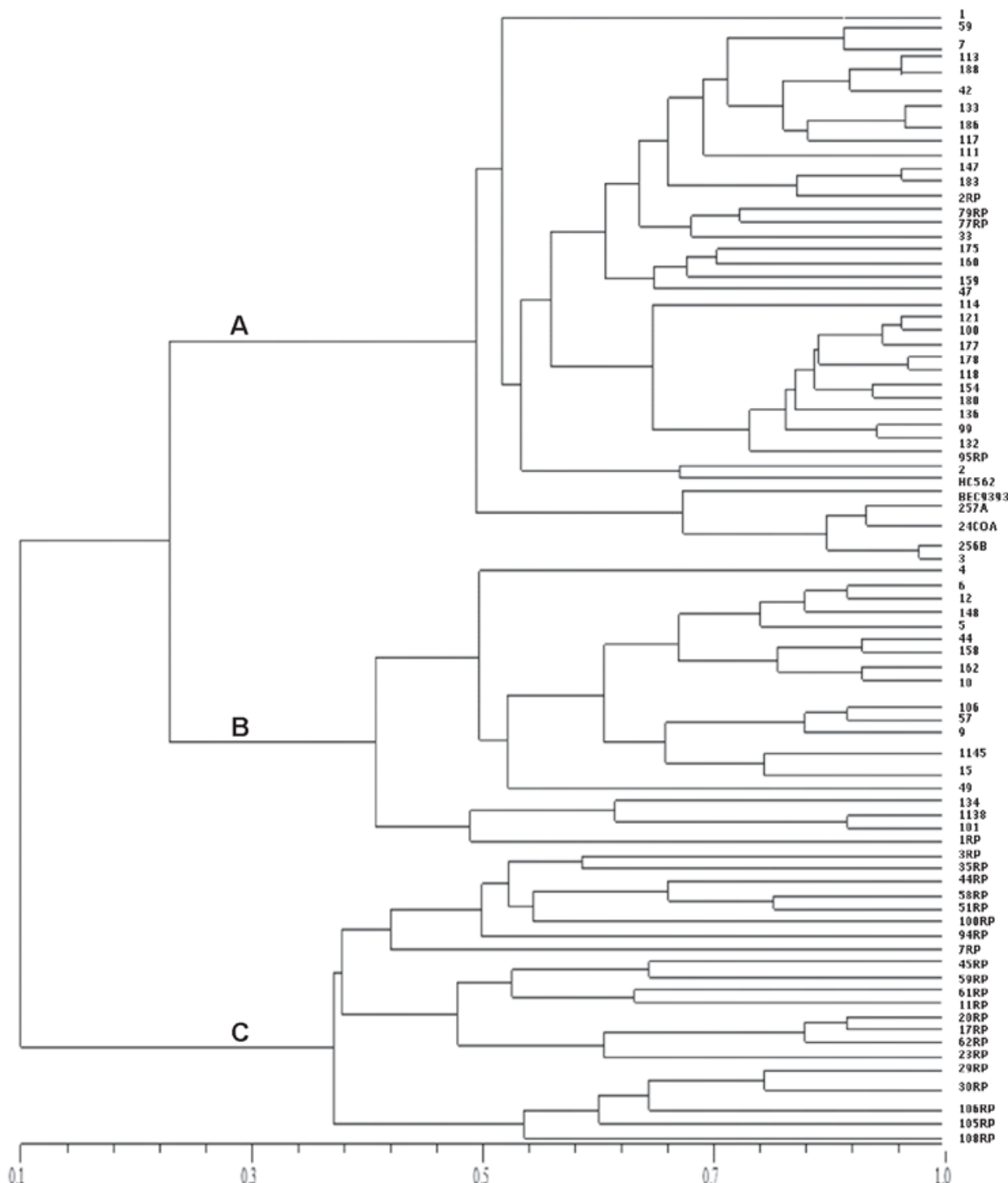

2. Meier P.A., Larter ᄂ.ע., wanlace ১.Ł., et aı. A proıngea outbreak of methicillin-resistant Staphylococcus aureus in the burn unit of a tertiary medical center. Infect Control Hosp Epidemiol 1996; $17: 798-802$.

3. Feil E.J., Smith J.M., Enright M.C., Spratt B.G. Estimating recombinational parameters in Streptococcus pneumoniae from multilocus sequence typing data. Genetics 2000;154:1439-50.

4. Feil E.J., Maiden M.C., Achtman M., Spratt B.G. The relative contributions of recombination and mutation to the divergence of clones of Neisseria meningitidis. Mol Biol Evol 1999; $16: 1496-1502$

5. Suerbaum S., Smith J. M., Bapumia K., et al. Free recombination within Helicobacter pylori. Proc Natl. Acad Sci 1998;13:12619-24.

6. Feil E.J., Holmes E.C., Bessen D.E., et al. Recombination within natural populations of pathogenic bacteria: short-term empirical estimates and long-term phylogenetic consequences. Proc Natl Acad Sci 2001;2:182-7.

7. Dominguez M.A.H., de Lencastre J., Tomasz A. Spread and maintenance of a dominant methicillin-resistant Staphylococcus aureus (MRSA) clone during an outbreak of MRSA disease in a Spanish hospital. J Clin Microbiol 1994;32:2081-7.
૪. Aıres ae sousa Ivı., ১ancnes 1. ১., van Beıkum A., et al. Characterization of methicillin-resistant Staphylococcus aureus isolates from Portuguese hospitals by multiple genotyping methods. Microb Drug Resist 1996;2:331-41.

9. Mato R., Santos Sanches I., Venditti M., et al. Spread of the multiresistant Iberian clone of methicillin resistance Staphylococcus aureus (MRSA) to Italy and Scotland. Microb Drug Resist 1998;4:107-12.

10. Rubin R.J., Harrington C.A., Poon A., et al. "The economic impact of Staphylococcus aureus infection in New York City hospitals." Emerg Infect Dis 1999;5:9-17.

11. Teixeira L., Resende C.A., Ormonde L.R., et al. Geographic spread of epidemic multiresistant Staphylococcus aureus clone in Brazil. J Clin Microbiol 1995;33:2400-4.

12. Melter O., Santos Sanches I., Schindler J., et al. Methicillin-resistant Staphylococcus aureus clonal types in the Czech Republic. J Clin Microbiol 1999;37:2798-2803.

13. Sanches I.S., Aires de Sousa M., Cleto L., et al. Tracing the origin of an outbreak of methicillin-resistant Staphylococcus aureus infections in a Portuguese hospital by molecular fingerprinting methods. Microb Drug Resist 1996;2:319-29. 
14. Coimbra M.V.D.S., Teixeira L.A., Ramos R.L.B., et al. Spread of the Brazilian epidemic clone of a multiresistant MRSA in two cities in Argentina J Med Microbiol 2000;49:187-92.

15. Sousa M.A., Sanches I.S., Ferro M.L., et al. Intercontinental spread of a multidrug-resistant methicillin-resistant Staphylococcus aureus clone. J Clin Microbiol 1998;36:2590-6.

16. dos Santos Soares M.J., da Silva-Carvalho M.C., Ferreira-Carvalho B.T., Figueiredo A.M.S. Spread of methicillin-resistant Staphylococcus aureus belonging to the Brazilian epidemic clone in a general hospital and emergence of heterogeneous resistance to glycopeptide antibiotics among these isolates. J Hosp Infect 2000;44:301-8.

17. Soares M.J.S., Teixeira L.A., Nunes M.R., et al. Analysis of different molecular methods for typing methicillin-resistant Staphylococcus aureus isolates belonging to the Brazilian epidemic clone. J Med Microbio 2001;50:732-42.

18. Branchini M.L.M., Morthland V.H., Tresoldi A.T. 1993 Application of genomic DNA subtyping by pulsed field gel electrophoresis and restriction enzyme analysis of plasmid DNA to characterize methicillin-resistant Staphylococcus aureus from two nosocomial outbreaks. Diagn Microbiol Infect Dis 2001; 17:275-81.

19. Enright M.C., Day N.P., Davies C.E., et al. 2000 Multilocus sequence typing for characterization of methicillin- resistant and methicillin-susceptible clones of Staphylococcus aureus. J Clin Microbiol 2001;38:1008-15.
20. Padoveze M.C., Tresoldi A.T., von Nowakonski A., et al. Nasal MRSA colonization of AIDS Patients cared for in a Brazilian university hosp Infect Control Hosp Epidemiol 2001; 22:783-5.

21. Sambrook J., Fritsch E.F., Maniatis T. Molecular cloning: a laboratory manual. New York Cold Spring Harbour 1989.

22. Ausubel F.M., Brente R., Kingston R.E., et al. Current Protocols in Molecular Biology. Green Publishing Associates, Brooklyn, NY 1988.

23. Koreen L., Ramaswamy S.V., Graviss E.A., et al. spa typing method for discriminating among Staphylococcus aureus isolates: implications for use of a single marker to detect genetic microand macrovariation, J Clin Microbiol 2004;42:792-9.

24. Montesinos I., Salido E., Delgado T., et al. Epidemiologic Genotyping of Methicillin-Resistant Staphylococcus aureus by Pulsed-Field Gel Electrophoresis at a University Hospital and Comparison with Antibiotyping and Protein A and Coagulase Gene Polymorphisms. J Clin Microbiol 2002;40:2119-25.

25. Diep B.A., Perdreau-Remington F., Sensabaugh F. 2003 Clonal characterization of Staphylococcus aureus by Multilocus Restriction Fragment Typing, a rapid screening approach for molecular epidemiology. J Clin Microbiol 2004;41:4559-64.

26. Rohlf F.J. NTSYS-PC 2.1. Numerical taxonomy and multivariate analysis system. Exeter Software, Setauket, NY.24 1997.

27. Kuhn G., Francioli P., Blanc D.S. Evidence for Clonal Evolution among Highly Polymorphic Genes in Methicillin-Resistant Staphylococcus aureus. J Bacteriol 2006;188:169-718. 